\title{
Geometric techniques for implicit two-dimensional systems *
}

\author{
Lorenzo Ntogramatzidis ${ }^{\star} \quad$ Michael Cantoni ${ }^{\dagger}$ \\ ${ }^{\star}$ Department of Mathematics and Statistics, \\ Curtin University, Perth, 6845 WA, Australia. \\ E-mail: L.Ntogramatzidis@curtin.edu.au \\ $\dagger$ Department of Electrical and Electronic Engineering, \\ The University of Melbourne, Parkville, 3010 VIC, Australia. \\ E-mail: cantoni@unimelb.edu.au
}

\begin{abstract}
Geometric tools are developed for two-dimensional (2-D) models in an implicit FornasiniMarchesini form. In particular, the structural properties of controlled and conditioned invariance are defined and studied. These properties are investigated in terms of quarter-plane causal solutions of the implicit model given compatible boundary conditions. The definitions of controlled and conditioned invariance introduced, along with the corresponding output-nulling and input-containing subspaces, are shown to be richer than the one-dimensional counterparts. The analysis carried out in this paper establishes necessary and sufficient conditions for the solvability of 2-D disturbance decoupling problems and unknown-input observation problems. The conditions obtained are expressed in terms of output-nulling and input-containing subspaces, which can be computed recursively in a finite number of steps.
\end{abstract}

Keywords: Two-dimensional systems; Implicit Fornasini-Marchesini models; Controlled and Conditioned Invariance.

\section{Introduction}

The notions of controlled and conditioned invariance underpin geometric control theory [2]. For one-dimensional (1-D) systems governed by the regular linear time-invariant state-space model

$$
\begin{array}{r}
x_{k+1}=A x_{k}+B u_{k} \\
y_{k}=C x_{k}+D u_{k}
\end{array}
$$

controlled invariant subspaces - also known as $(A, B)$-invariants - are the subspaces satisfying the inclusion $A \mathcal{V} \subseteq \mathcal{V}+\operatorname{im} B$. These subspaces have the fundamental system-theoretic interpretation

\footnotetext{
*This work was partially supported by the Australian Research Council (DP0986577 and FT12010060).
} 
of being the locii of trajectories of (1), in the following sense: (a) if the initial state $x_{0}$ lies on a controlled invariant subspace $\mathcal{V}$, a control $u_{k}$ exists that maintains the entire state trajectory on $\mathcal{V}$; and conversely (b) if for any initial condition on a subspace $\mathcal{L}$ the entire trajectory can be kept on $\mathcal{L}$ with a suitable control function, $\mathcal{L}$ is controlled invariant. In the standard $1-\mathrm{D}$ context, controlled invariance also enjoys a fundamental feedback property; i.e. (c) the control input that maintains the state trajectory on a controlled invariant subspace can always be expressed in terms of a static state feedback input $u_{k}=F x_{k}$. In other words, the subspace $\mathcal{V}$ is controlled invariant if, and only if, a feedback matrix $F$ exists such that $\mathcal{V}$ is $(A+B F)$-invariant. In several classical references this property is used as the very definition of controlled invariance, see e.g. [23].

Conditioned invariance for 1-D systems - also referred to as $(C, A)$-invariance - is the dual concept of controlled invariance. A conditioned invariant subspace is defined as a subspace $\mathcal{S}$ satisfying $A(\mathcal{S} \cap \operatorname{ker} C) \subseteq \mathcal{S}$. The system-theoretic interpretation of these subspaces usually lies in the context of unknown-input observations. Loosely, conditioned invariant subspaces represent the part of the state vector of (1-2) that cannot be reconstructed using observers that have access to $y_{k}$ but not to $u_{k}$, see e.g. [22] and [21, Ch. 5].

Over the last forty years, controlled and conditioned invariant subspaces have played a role in the solution of a number of control and estimation problems, including disturbance decoupling, unknowninput observation, model matching, fault detection, non-interaction, and optimal control/filtering problems; see e.g. the monographs $[23,2,21]$ and the references cited therein. For this reason, various efforts have been devoted to extend the notion of controlled invariance to 2-D systems, as discussed further below.

In [5], a first definition of 2-D controlled invariance was provided for the regular first-order form of the Fornasini-Machesini (FM) model [10]

$$
x_{i+1, j+1}=A_{1} x_{i+1, j}+A_{2} x_{i, j+1}+B_{1} u_{i+1, j}+B_{2} u_{i, j+1} .
$$

Given 'south-west' boundary conditions with values taken from such an invariant subspace $\mathcal{V}$, the definition guarantees that a control input can be found to maintain the trajectory generated by (3) on the subspace $\mathcal{V}$; indeed, such a control input can be expressed as a static local-state feedback, $u_{i, j}=F x_{i, j}$. However, a control input that maintains the solution of (3) on a subspace $\mathcal{L}$, for any $\mathcal{L}$-valued boundary condition, may exist without $\mathcal{L}$ necessarily satisfying the definition of controlled invariance given in [5]. So while this definition enjoys good feedback properties, as recently explored in [20], it does not univocally characterise the set of trajectories generated by (3). As a consequence, when it is used in the solution of decoupling, control and estimation problems, the definition can only lead to sufficient - and hence potentially conservative - conditions, see Remark 3.2 in [5].

A second definition of controlled invariance for 2-D systems was provided in [12] for the implicit 2-D model

$$
E x_{i+1, j+1}=A_{1} x_{i+1, j}+A_{2} x_{i, j+1}+B u_{i, j} .
$$


The motivation for considering this model form was to characterise the solutions of an implicit Roesser model over a bounded frame. The drawback of this model is the lack of a static feedback characterisation of controlled invariance, since the form of the model (4) is not closed under the static feedback control $u_{i, j}=F x_{i, j}$. Hence, a feedback property as the one discussed for 1-D controlled invariance has no meaning in this case. To combine the advantages of the two aforementioned definitions, without incurring in the corresponding drawbacks, [17] proposes and studies a 2-D counterpart of controlled invariance in term of the original regular form of the FM model [9]

$$
x_{i+1, j+1}=A_{0} x_{i, j}+A_{1} x_{i+1, j}+A_{2} x_{i, j+1}+B u_{i, j} .
$$

As is the case for (3), this 2-D model, along with the output equation $y_{i, j}=C x_{i, j}+D u_{i, j}$, can realise any bivariate proper rational transfer function, see [9]. However, by contrast with the model (3), the input now appears only once. As such, the form of this model is closed under static localstate feedback $u_{i, j}=F x_{i, j}$. Moreover, unlike the form of (3), its dual is also well-defined, so that conditioned invariance can be introduced in a natural way, as discussed later.

In [17], it was shown that the definition of controlled invariance in [12] can be extended to models in the form (5). This definition retains the fundamental properties listed as (a) and (b) in the discussion of the 1-D case above. As for systems over rings [11], it was also shown in [17] that the notion of controlled invariance introduced for 2-D systems is richer than its 1-D counterpart; in the 2-D case there is a need to distinguish between controlled invariant subspaces, as loci of solutions of (5) generally, from the subset of those for which the associated control input can be expressed as a static local state feedback. These latter are subsequently referred to as controlled invariants of feedback type. The definition, compared to the one in [5], characterises univocally and in finite terms the subspaces of trajectories of a 2-D system that are generated by static local-state feedback controls, leading to necessary and sufficient conditions for the solution of disturbance decoupling problems, thereby eliminating the potential conservatism of existing results in [5, 20], which are based on sufficient conditions.

In [19] it was shown how the geometric setting in [17] could be adapted to strictly proper FM models in implicit form. The first aim of this paper is to extend the framework developed in [19] to the following implicit model form with non-zero feed-through term $D$ :

$$
E x_{i+1, j+1}=A_{0} x_{i, j}+A_{1} x_{i+1, j}+A_{2} x_{i, j+1}+B u_{i, j} ; \quad y_{i, j}=C x_{i, j}+D u_{i, j}
$$

This implicit model can be used realise bivariate rational transfer functions in a way that involves a smaller semi-state dimension than other model classes, while retaining the property of recursive computability [24]. Furthermore, the introduction of the possibly singular matrix $E$ can be used to capture algebraic constraints between the local state and the control variables, given admissible 'south-west' boundary conditions. The second goal of the paper is to show that the new notions of 2-D controlled invariance and 2-D controlled invariance of feedback type give rise to a complete solution of the classic disturbance decoupling problem. In particular, the solution is characterised in 
terms of constructive necessary and sufficient conditions. The problem of parameterising the set of static local-state feedback controls, $u_{i, j}=F x_{i, j}$, which generate solutions of (5) that lie on controlled invariant subspaces of feedback type is then investigated. The dual notion, called 2-D conditioned invariance of output-injection type, is also considered.

Notation: Throughout, we denote by $\mathbb{Z}$ and $\mathbb{N}$ the integers, and positive integers including zero (i.e., natural numbers), respectively. The image and null-space of a linear operator $M$ are denoted by $\operatorname{im} M$ and $\operatorname{ker} M$, respectively. For convenience, a linear mapping between finite-dimensional spaces and a matrix representation with respect to a particular basis are not distinguished notationally. The Moore-Penrose pseudo-inverse of $M$ is denoted with $M^{\dagger}$. Given $A: \mathbb{R}^{n} \rightarrow \mathbb{R}^{m}$ and a subspace $\mathcal{Y} \subseteq \mathbb{R}^{m}$, we denote by $A^{-1} \mathcal{Y}$ the set $\left\{x \in \mathbb{R}^{n} \mid A x \in \mathcal{Y}\right\}$. When $A$ is invertible, the notation $A^{-1}$ is also used to denote the inverse mapping and the inverse matrix representation. Subspaces are denoted by calligraphic letters. The annihilator of a subspace $\mathcal{S}$ of the linear space $\mathcal{X}$ is denoted by $\mathcal{S}^{\perp}=\left\{x \in \mathcal{X} \mid x^{\mathrm{T}} s=0 \forall s \in \mathcal{S}\right\}$; the annihilator resides in the dual space of $\mathcal{X}$.

\section{Invariant Subspaces for Implicit FM Models}

Consider the implicit FM model $[9,8]$

$$
\begin{aligned}
E x_{i+1, j+1} & =A_{0} x_{i, j}+A_{1} x_{i+1, j}+A_{2} x_{i, j+1}+B u_{i, j}, \\
y_{i, j} & =C x_{i, j}+D u_{i, j}
\end{aligned}
$$

where, for all $i, j \in \mathbb{Z}$, the vector $x_{i, j} \in \mathcal{X}=\mathbb{R}^{n}$ is the latent variable, $u_{i, j} \in \mathcal{U}=\mathbb{R}^{m}$ is the input and $y_{i, j} \in \mathcal{Y}=\mathbb{R}^{p}$ is the output. Hence, $E, A_{0}, A_{1}, A_{2} \in \mathbb{R}^{q \times n}, B \in \mathbb{R}^{q \times m}$ and $C \in \mathbb{R}^{p \times n}$. The dimension of the outer space, denoted by $\underline{\mathcal{X}}=\mathbb{R}^{q}$, equals the number of equations in (6). The special feature of the implicit model is that the matrices $E, A_{0}, A_{1}, A_{2}$ are in general not square; and when square (i.e., if $q=n$ ), these may be singular. We identify the system (6-7) with the septuple $\Sigma \stackrel{\text { def }}{=}\left(E ; A_{0}, A_{1}, A_{2} ; B ; C ; D\right)$. If $D$ is the zero matrix, system (6-7) is said to be strictly proper.

We are ultimately interested in the (eventually controlled) evolution of quarter-plane causal solutions of (6) over $\mathbb{N} \times \mathbb{N}$, given suitable 'south-west' boundary conditions on $x_{i, j}$ for $(i, j) \in \mathfrak{B}_{0}$, where

$$
\mathfrak{B}_{k} \stackrel{\text { def }}{=}(\{k\} \times\{j \in \mathbb{Z} \mid j \geq k\}) \cup(\{j \in \mathbb{Z} \mid j \geq k\} \times\{k\}), \quad k \in \mathbb{Z}
$$

In particular, we consider the evolutions of the latent variable $x_{i, j}$ over the region

$$
\mathfrak{B} \stackrel{\text { def }}{=} \bigcup_{k=0}^{\infty} \mathfrak{B}_{k}=\mathbb{N} \times \mathbb{N},
$$

such that $\left\{x_{i, j} \mid(i, j) \in \mathfrak{B}_{k}\right\}$ is completely determined by $\left\{u_{i, j} \mid(i, j) \in \bigcup_{l=0}^{k-1} \mathfrak{B}_{l}\right\}$ and the boundary conditions over $\mathfrak{B}_{0}$, for every $k>0$. 


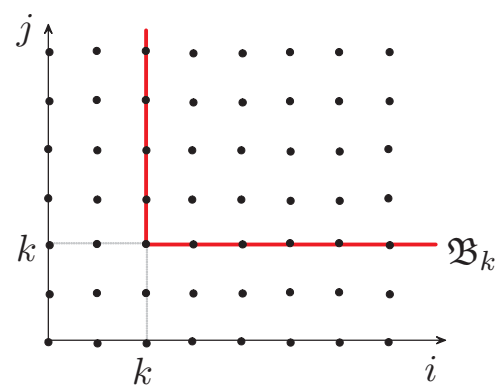

Figure 1: Boundary conditions and sets $\mathfrak{B}_{k}$.

Given a subspace $\mathcal{W}$ of $\mathcal{X}$, we say that (6) has a $\mathcal{W}$-valued boundary condition if $x_{i, j} \in \mathcal{W}$ for all $(i, j) \in \mathfrak{B}_{0}$; on the other hand, given an input over $\mathfrak{B},(6)$ is said to admit a $\mathcal{W}$-valued trajectory if there is a solution such that $x_{i, j} \in \mathcal{W}$ for all $(i, j) \in \mathfrak{B}$.

Definition 2.1 A subspace $\mathcal{J} \subseteq \mathcal{X}$ is called invariant for the quadruple $\left(E ; A_{0}, A_{1}, A_{2}\right)$ if

$$
A_{i} \mathcal{J} \subseteq E \mathcal{J}, \quad i \in\{0,1,2\}
$$

Remark 2.1 Notice that (8) is equivalent to

$$
\sum_{i=0}^{2}\left(A_{i} \mathcal{J}\right) \subseteq E \mathcal{J} .
$$

Invariant subspaces are important for implicit systems because they can be used in the investigation of (6) for zero-input and compatible boundary conditions; i.e. boundary conditions $\left\{x_{i, j} \in\right.$ $\left.\mathcal{X} \mid(i, j) \in \mathfrak{B}_{0}\right\}$ for which (6) admits a solution $\left\{x_{i, j} \in \mathcal{X} \mid(i, j) \in \mathfrak{B}\right\}$ with $u_{i, j}=0$ for all $(i, j) \in \mathfrak{B}$. The following lemma shows the relation between the concept of invariance for $\left(E ; A_{0}, A_{1}, A_{2}\right)$ defined here and the existence of zero input solutions for $\Sigma$.

Lemma 2.1 A subspace $\mathcal{J}$ of $\mathcal{X}$ is $\left(E ; A_{0}, A_{1}, A_{2}\right)$-invariant if, and only if, (6) admits a solution $\left\{x_{i, j} \in \mathcal{J} \mid(i, j) \in \mathfrak{B}\right\}$ for arbitrary $\mathcal{J}$-valued boundary conditions and zero input.

Proof: Suppose $\mathcal{J}$ is invariant for $\left(E ; A_{0}, A_{1}, A_{2}\right)$. Given arbitrary $\mathcal{J}$-value boundary conditions we then have $x_{0,0}, x_{1,0}$ and $x_{0,1}$ in $\mathcal{J}$ and by virtue of $(9)$, a vector $x_{1,1} \in \mathcal{J}$ exists such that

$$
E x_{1,1}=A_{0} x_{0,0}+A_{1} x_{1,0}+A_{2} x_{0,1} .
$$

By repeating this process for all vectors of the $\mathcal{J}$-valued boundary condition, we have constructed a solution for (6) comprising only vectors in $\mathcal{J}$ for $(i, j) \in \mathfrak{B}_{1}$. Continuing recursively in this manner, yields a solution for $(i, j) \in \mathfrak{B}_{k}$, for $k \geq 0$. Conversely, suppose to the contrapositive that there exists $x_{0,0}, x_{1,0}, x_{1,0}$ in $\mathcal{J}$ for which $A_{0} x_{1,0}+A_{1} x_{1,0}+A_{2} x_{0,1} \notin E \mathcal{J}$. Then clearly, a solution of (6) with $x_{1,1} \in \mathcal{J}$ cannot exist with the input set to zero. As such, (9) necessarily holds if (6) has $\mathcal{J}$-valued trajectory for arbitrary $\mathcal{J}$-valued boundary conditions and input set to zero. 
The following provides another useful way to see that there exists a $\mathcal{J}$-valued solution of (6) with zero input whenever $\mathcal{J}$ is $\left(E ; A_{0}, A_{1}, A_{2}\right)$-invariant. Given a basis matrix $J$ of $\mathcal{J}$, three matrices $X_{0}, X_{1}, X_{2}$ exist such that

$$
A_{i} J=E J X_{i}, \quad i \in\{0,1,2\}
$$

Now, consider a $\mathcal{J}$-valued boundary condition so that for each $(i, j) \in \mathfrak{B}_{0}$ we can write $x_{i, j}=J \xi_{i, j}$ for some $\xi_{i, j}$. For any $(i, j) \in \mathfrak{B}_{0}$, we have $(i+1, j),(i, j+1) \in \mathfrak{B}_{0}$ and in view of $(10)$,

$$
\begin{aligned}
E x_{i+1, j+1} & =A_{0} J \xi_{i, j}+A_{1} J \xi_{i+1, j}+A_{2} J \xi_{i, j+1} \\
& =E J X_{0} \xi_{i, j}+E J X_{1} \xi_{i+1, j}+E J X_{2} \xi_{i, j+1} .
\end{aligned}
$$

Now, define the regular (i.e. explicit) FM model

$$
\xi_{i+1, j+1}=X_{0} \xi_{i, j}+X_{1} \xi_{i+1, j}+X_{2} \xi_{i, j+1} .
$$

By direct substitution one can verify that $x_{i, j}=J \xi_{i, j}$ satisfies (6) with zero input for $(i, j) \in \mathfrak{B}_{1}$; this is clearly $\mathcal{J}$-valued. Continuing recursively, yields a $\mathcal{J}$-valued solution over $\mathfrak{B}$. Note that the preceding analysis does not imply all solutions of (6) with zero input and given $\mathcal{J}$-valued boundary condition are necessarily $\mathcal{J}$-valued, nor that $\mathcal{J}$-valued solutions are unique.

Theorem 2.1 Suppose $\mathcal{J}$ is $\left(E ; A_{0}, A_{1}, A_{2}\right)$-invariant. A $\mathcal{J}$-valued solution of (6) with zero input is unique if, and only if, $\operatorname{ker} E \cap \mathcal{J}=\{0\}$.

Proof: Consider a $\mathcal{J}$-valued boundary condition. Then $x_{0,0}, x_{1,0}, x_{0,1} \in \mathcal{J}$. Since $\mathcal{J}$ is invariant for $\left(E ; A_{0}, A_{1}, A_{2}\right)$, there exists a vector $\xi_{0}$ such that

$$
\hat{x} \stackrel{\text { def }}{=} A_{0} x_{0,0}+A_{1} x_{1,0}+A_{2} x_{0,1}=E J \xi_{0}
$$

where $J$ is a basis matrix for $\mathcal{J}$. The set of such vectors $\xi_{0}$ is parametrised in the null-space of $E J$ as $\xi_{0}=(E J)^{\dagger} \hat{x}+v_{1}$ with $v_{1} \in \operatorname{ker}(E J)$. The set of all vectors $x_{1,1}$ that solve $E x_{1,1}=E J \xi_{0}$ is given by $x_{1,1}=J \xi_{0}+v_{2}$, where $v_{2} \in$ ker $E$. Thus, the set of all possible $x_{1,1}$ compatible with the assigned boundary condition is given by $x_{1,1}=J(E J)^{\dagger} \hat{x}+J v_{1}+v_{2}$, where $v_{2} \in \operatorname{ker}(E)$ and $v_{1} \in \operatorname{ker}(E J)$. The term $J v_{1}$ is a vector of ker $E \cap \mathcal{J}$, since trivially $J \operatorname{ker}(E J)=\operatorname{ker} E \cap \mathcal{J}$. Consider a basis matrix $\left[\begin{array}{ll}U_{1} & U_{2}\end{array}\right]$ of ker $E$ such that $U_{1}$ is a basis of ker $E \cap \mathcal{J}$, whereby $x_{1,1}$ can be expressed in terms of two vectors $\alpha$ and $\beta$ as

$$
x_{1,1}=J(E J)^{\dagger} \hat{x}+J v_{1}+U_{1} \alpha+U_{2} \beta
$$

If ker $E \cap \mathcal{J}=\{0\}$, then $x_{1,1}=J(E J)^{\dagger} \hat{x}+U_{2} \beta$. Since we are considering a $\mathcal{J}$-valued solution and $\operatorname{im} U_{2} \cap \mathcal{J}=\{0\}$, it follows that $\beta=0$. Thus, $x_{1,1}=J(E J)^{\dagger} \hat{x}$ is the unique value in this case. The same argument can be repeated recursively, to obtain the unique $x_{i, j}$, given $\mathcal{J}$-valued $x_{i-1, j-1}, x_{i-1, j}, x_{i, j-1}$ for all $(i, j) \in \mathfrak{B}_{k}, k \geq 1$.

Conversely, when ker $E \cap \mathcal{J} \neq\{0\}$, it can be seen from (11) that there is freedom in the choice of solution. Hence, $\operatorname{ker} E \cap \mathcal{J}=\{0\}$ holds necessarily if a $\mathcal{J}$-valued solution is unique. 
The issue of the uniqueness for implicit FM models was considered in [6], where a sufficient condition was proposed based on a classic 1-D result [3]. The condition given in Theorem 2.1 is stated in terms of a necessary and sufficient condition. Notice that the sufficiency in Theorem 2.1 can also be proved by noticing that if $\operatorname{ker}(E J) \neq\{0\}$, given a $\mathcal{J}$-valued boundary condition one can construct a solution $x_{i, j}=J \xi_{i, j}$, where $\xi_{i, j}$ is the solution of the recursive difference equation

$$
\xi_{i+1, j+1}=X_{0} \xi_{i, j}+X_{1} \xi_{i+1, j}+X_{2} \xi_{i, j+1}+v_{i, j}
$$

where for all $(i, j) \in \mathfrak{B}$ the vector $v_{i, j}$ lies on $\operatorname{ker}(E J)$. It is easily seen that $x_{i, j}=J \xi_{i, j}$ is indeed a solution of (6) for all $v_{i, j} \in \operatorname{ker}(E J)$. Therefore, the solution is not unique, but rather parametrised by arbitrary vectors in $\operatorname{ker}(E J)$.

The set of all $\left(E ; A_{0}, A_{1}, A_{2}\right)$-invariant subspaces is closed under subspace addition. Therefore, it admits a maximum element, which is given by the sum of all invariant subspaces of $\left(E ; A_{0}, A_{1}, A_{2}\right)$. This subspace, herein denoted by $\mathcal{J}^{\star}$, can be computed by means of the following lemma.

Lemma 2.2 The subspace $\mathcal{J}^{\star}$ can be computed as the last term of the monotonically non-increasing sequence of subspaces $\left\{\mathcal{J}_{i}\right\}_{i \in \mathbb{N}}$ given by

$$
\left\{\begin{array}{l}
\mathcal{J}_{0}=\mathcal{X} \\
\mathcal{J}_{i}=\bigcap_{j=0}^{2}\left(A_{j}^{-1} E \mathcal{J}_{i-1}\right) \cap \mathcal{J}_{i-1} \quad i \in\{1,2, \ldots, k\},
\end{array}\right.
$$

where the integer $k \leq n-1$ is determined by the condition $\mathcal{J}_{i+1}=\mathcal{J}_{i}\left(=\mathcal{J}^{\star}\right)$.

Proof: First, we show by induction that the sequence $\left\{\mathcal{J}_{i}\right\}_{i \in \mathbb{N}}$ is monotonically non-increasing. To this end, notice that $\mathcal{J}_{0} \supseteq \mathcal{J}_{1}$. Suppose $\mathcal{J}_{h-1} \supseteq \mathcal{J}_{h}$, and let us show that $\mathcal{J}_{h} \supseteq \mathcal{J}_{h+1}$. From $\mathcal{J}_{h-1} \supseteq \mathcal{J}_{h}$ we get

$$
\mathcal{J}_{h}=\bigcap_{j=0}^{2}\left(A_{j}^{-1} E \mathcal{J}_{h-1}\right) \cap \mathcal{J}_{h-1} \supseteq \bigcap_{j=0}^{2}\left(A_{j}^{-1} E \mathcal{J}_{h}\right) \cap \mathcal{J}_{h}
$$

Hence, $\mathcal{J}_{h}=\mathcal{J}_{h+1}$. Now, we show that $\mathcal{J}^{\star}$ is invariant for $\left(E ; A_{0}, A_{1}, A_{2}\right)$. For $\mathcal{J}^{\star}$ there holds

$$
\mathcal{J}^{\star}=\bigcap_{j=0}^{2}\left(A_{j}^{-1} E \mathcal{J}^{\star}\right) \cap \mathcal{J}^{\star}
$$

Let $h \in\{0,1,2\}$. Applying $A_{h}$ to both sides of (12) we get

$$
\begin{aligned}
A_{h} \mathcal{J}^{\star} & =A_{h}\left(\bigcap_{j=0}^{2}\left(A_{j}^{-1} E \mathcal{J}^{\star}\right) \cap \mathcal{J}^{\star}\right) \subseteq \bigcap_{j=0}^{2}\left(A_{h} A_{j}^{-1} E \mathcal{J}^{\star}\right) \\
& \subseteq\left(A_{h} A_{h}^{-1} E \mathcal{J}^{\star}\right) \subseteq E \mathcal{J}^{\star} \cap \operatorname{im} A_{h} \subseteq E \mathcal{J}^{\star} .
\end{aligned}
$$

Since this is true for any $h \in\{0,1,2\}$, then $\mathcal{J}^{\star}$ is invariant for $\left(E ; A_{0}, A_{1}, A_{2}\right)$. 
Now, we show that $\mathcal{J}^{\star}$ is the largest invariant for $\left(E ; A_{0}, A_{1}, A_{2}\right)$. Let $\overline{\mathcal{J}}$ be another invariant for $\left(E ; A_{0}, A_{1}, A_{2}\right)$, so that $A_{i} \overline{\mathcal{J}} \subseteq E \overline{\mathcal{J}}$ implies $\overline{\mathcal{J}} \subseteq A_{i}^{-1} E \overline{\mathcal{J}}$. It follows that

$$
\overline{\mathcal{J}} \subseteq \bigcap_{j=0}^{2}\left(A_{j}^{-1} E \overline{\mathcal{J}}\right)
$$

Now we show that every subspace of the sequence $\left\{\mathcal{J}_{i}\right\}_{i \in \mathbb{N}}$ contains $\overline{\mathcal{J}}$, so that, in particular, $\mathcal{J}^{\star} \supseteq \overline{\mathcal{J}}$. Clearly, $\mathcal{J}_{0} \supseteq \overline{\mathcal{J}}$. Suppose $\mathcal{J}_{i} \supseteq \overline{\mathcal{J}}$. Therefore

$$
\mathcal{J}_{i+1}=\bigcap_{j=0}^{2}\left(A_{j}^{-1} E \mathcal{J}_{i}\right) \cap \mathcal{J}_{i} \supseteq \bigcap_{j=0}^{2}\left(A_{j}^{-1} E \overline{\mathcal{J}}\right) \cap \overline{\mathcal{J}} \supseteq \overline{\mathcal{J}}
$$

in view of $(13)$. Hence, $\mathcal{J}^{\star} \supseteq \overline{\mathcal{J}}$.

\section{Controlled invariance}

In order to study quarter-plane causal solutions of the implicit FM model (6) for (south-west) boundary conditions on $\mathfrak{B}_{0}$ and appropriately selected control input, we introduce the notion of controlled invariance.

Definition 3.1 A subspace $\mathcal{V}$ of $\mathcal{X}$ is a controlled invariant subspace for $\Sigma$ if

$$
A_{i} \mathcal{V} \subseteq E \mathcal{V}+\operatorname{im} B, \quad i \in\{0,1,2\}
$$

The following theorem provides a system-theoretic interpretation of controlled invariance.

Theorem 3.1 For all $\mathcal{V}$-valued boundary conditions there exists a control input such that (6) admits a quarter-plane causal $\mathcal{V}$-valued trajectory if, and only if, $\mathcal{V}$ is a controlled invariant.

Proof: To establish sufficiency, suppose $\mathcal{V}$ is a controlled invariant subspace. We proceed inductively to construct a control input that yields a $\mathcal{V}$-valued trajectory which exhibits a quarter-plane causal dependence on the input. First note that given any $\mathcal{V}$-valued boundary condition, we have $x_{0,0}, x_{1,0}, x_{0,1} \in \mathcal{V}$, whereby controlled invariance implies the existence of an $x \in \mathcal{V}$ and $\omega \in \mathcal{U}$ such that

$$
A_{0} x_{0,0}+A_{1} x_{1,0}+A_{2} x_{0,1}=E x+B \omega .
$$

Let $u_{0,0}=-\omega$ and $x_{1,1}=x$. Similarly, for $(i+1, j+1) \in \mathfrak{B} \backslash \mathfrak{B}_{0}$, whenever $x_{i, j}, x_{i+1, j}, x_{i, j+1} \in \mathcal{V}$, controlled invariance implies existence of an $x \in \mathcal{V}$ and $\omega \in \mathcal{U}$ such that

$$
A_{0} x_{i, j}+A_{1} x_{i+1, j}+A_{2} x_{i, j+1}=E x+B \omega .
$$

Let $u_{i, j}=-\omega$ and $x_{i+1, j+1}=x$. Continuing in this fashion yields a quarter-plane causal trajectory that evolves according (6) over $\mathfrak{B}$. 
To prove necessity, suppose that (14) does not hold. Then there exist $x^{\prime}, x^{\prime \prime}, x^{\prime \prime \prime} \in \mathcal{V}$ such that no $x \in \mathcal{V}$ and $\omega \in \mathcal{U}$ can be found for which

$$
E x=A_{0} x^{\prime}+A_{1} x^{\prime \prime}+A_{2} x^{\prime \prime \prime}+B \omega .
$$

Therefore, choosing $x_{0,0}=x^{\prime}, x_{1,0}=x^{\prime \prime}, x_{0,1}=x^{\prime \prime \prime}$, we find that $(6)$ does not admit a $\mathcal{V}$-valued solution $x_{1,1}$ for any choice of input $u_{0,0}$, which would contradict the existence of an appropriate control input for $\mathcal{V}$-valued arbitrary boundary conditions.

Remark 3.1 In this paper we follow the classical geometric approach of [1] and [23]. This involves first defining controlled invariant subspaces in terms of a geometric subspace inclusion, and then identifying necessary and sufficient conditions to provide system-theoretic interpretations of these subspaces. The converse approach, which has been gaining popularity in recent years (see e.g. [21]), seeks to directly define controlled invariance and output-nulling subspaces in terms of the properties of the (semi-)state (and output) trajectories. Since the objective here is to provide a complete geometric picture for implicit 2-D systems, including the notion of conditioned invariance and input-containing subspaces, which are herein most simply defined via duality arguments, for the sake of consistency throughout the classical approach of [1] is employed.

As mentioned in the introduction, for the definitions of controlled invariance given in [4], [5] and [6], where a first-order FM model was considered, only the if part of the statement of Theorem 3.1 holds true. Necessity for the implicit model (6) considered here holds as in the regular case of this form studied in [17].

\subsection{Output-Nulling Subspaces}

In many control problems it is of interest to derive control laws that maintain certain outputs of a system at zero. The most famous example is the disturbance decoupling problem, [2]. This requirement leads to the notion of output-nulling subspace. An output-nulling subspace for $\Sigma$ is such that (6-7) have a $\mathcal{V}$-valued solution with an identically zero output for any $\mathcal{V}$-valued boundary condition. When the feedthrough matrix $D$ is zero, a solution of (6-7) yields zero output if and only if for all $(i, j) \in \mathfrak{B}$ the local state $x_{i, j}$ lies in ker $C$. Hence, for strictly proper systems an output-nulling subspace is simply a controlled invariant subspace contained in $\operatorname{ker} C$. When the feed-through is not zero, the definition is as follows.

Definition $3.2 \mathcal{V}$ is output-nulling for $\Sigma$ if

$$
\left[\begin{array}{ccc}
A_{0} & A_{1} & A_{2} \\
C & O & O
\end{array}\right] \bigoplus_{k=1}^{3} \mathcal{V} \subseteq(E \mathcal{V} \oplus\{0\})+\operatorname{im}\left[\begin{array}{l}
B \\
D
\end{array}\right]
$$

Theorem 3.2 A subspace $\mathcal{V}$ is output-nulling for $\Sigma$ if, and only if, there exists a control law such that (6-7) admits a $\mathcal{V}$-valued solution with zero output given an arbitrary $\mathcal{V}$-valued boundary condition. 
Proof: By virtue of Definition 3.2, if we consider $x_{i, j}, x_{i+1, j}, x_{i, j+1} \in \mathcal{V}$, then two vectors $\hat{x} \in \mathcal{V}$ and $\omega \in \mathcal{U}$ exist such that

$$
\begin{array}{rlrl}
A x_{i, j}+A_{1} x_{i+1, j}+A_{2} x_{i, j+1} & = & E \hat{x}+ & B \omega \\
C x_{i, j} & = & D \omega
\end{array}
$$

Using the same type of argument employed in the proof of Theorem 3.1, a control input can be recursively constructed such that (6-7) admits a $\mathcal{V}$-valued solution for which the output is identically zero. Necessity can be proved following the same lines of the proof of necessity in Theorem 3.1.

The set of output-nulling subspaces of (6-7) is denoted by $\mathcal{V}(\Sigma)$. As for the set of all controlled invariant subspaces, this set is closed under subspace addition but not under subspace intersection. Therefore, $(\mathcal{V}(\Sigma),+; \subseteq)$ is a (non-distributive and modular) upper semi-lattice with respect to the binary operation + and with respect to the partial ordering $\subseteq$. Thus, it admits a maximum $\mathcal{V}^{\star}$ given by the sum of all elements of $\mathcal{V}(\Sigma)$; i.e., $\mathcal{V}^{\star} \stackrel{\text { def }}{=} \max \mathcal{V}(\Sigma)=\sum_{\mathcal{V} \in \mathcal{V}(\Sigma)} \mathcal{V}$. The following lemma extends the well-known algorithm for the computation of $\mathcal{V}^{\star}$ introduced in [1].

Lemma 3.1 $\mathcal{V}^{\star}$ is the last term of the monotonically non-increasing sequence $\left\{\mathcal{V}_{i}\right\}_{i}$ given by

$$
\left\{\begin{array}{l}
\mathcal{V}_{0}=\mathcal{X} \\
\mathcal{V}_{i}=\bigcap_{j=1}^{2}\left[\begin{array}{c}
A_{j} \\
O
\end{array}\right]^{-1}\left(\left(E \mathcal{V}_{i-1} \oplus\{0\}\right)+\operatorname{im}\left[\begin{array}{c}
B \\
D
\end{array}\right]\right) \cap\left[\begin{array}{c}
A_{0} \\
C
\end{array}\right]^{-1}\left(\left(E \mathcal{V}_{i-1} \oplus\{0\}\right)+\operatorname{im}\left[\begin{array}{c}
B \\
D
\end{array}\right]\right)
\end{array}\right.
$$

where $i \in\{1,2, \ldots, k\}$ and where the integer $k \leq n-1$ is determined by the condition $\mathcal{V}_{k+1}=\mathcal{V}_{k}$; i.e., $\mathcal{V}^{\star}=\mathcal{V}_{k}$.

Proof: The proof of this result follows the same lines of that of Lemma 2.2; see also the proof of [17, Lemma 4.1].

Remark 3.2 When $D=0$, the sequence of subspaces $\left\{\mathcal{V}_{i}\right\}_{i}$ given in Lemma 3.1 reduces to the following sequence:

$$
\left\{\begin{array}{l}
\mathcal{V}_{0}=\mathcal{X} \\
\mathcal{V}_{i}=\bigcap_{j=0}^{2} A_{j}^{-1}\left(E \mathcal{V}_{i-1}+\operatorname{im} B\right) \cap \operatorname{ker} C \quad i \in\{1,2, \ldots, k\}
\end{array}\right.
$$

\subsection{Controlled Invariants of Feedback Type}

By contrast with the model considered in [12], the FM model (6-7) is closed under the feedback $u_{i, j}=F x_{i, j}$, which gives rise to the closed-loop local state update equation

$$
E x_{i+1, j+1}=\left(A_{0}+B F\right) x_{i, j}+A_{1} x_{i+1, j}+A_{2} x_{i, j+1} .
$$

It is easy to see that, differently from 1-D systems, the notion of controlled invariance alone is not sufficient to guarantee the existence of a feedback matrix $F$ that maintains the local state $x_{i, j}$ on a controlled invariant subspace $\mathcal{V}$ for $\mathcal{V}$-valued boundary conditions. For this reason, we introduce the concept of controlled invariance of feedback type. 
Definition 3.3 A subspace $\mathcal{W}$ is controlled-invariant of feedback type for $\Sigma$ if

- $A_{0} \mathcal{W} \subseteq E \mathcal{W}+\operatorname{im} B$;

- $A_{1} \mathcal{W}+A_{2} \mathcal{W} \subseteq E \mathcal{W}$.

Notice that $A_{1} \mathcal{W}+A_{2} \mathcal{W} \subseteq E \mathcal{W}$ is equivalent to $A_{i} \mathcal{W} \subseteq E \mathcal{W}$ for $i \in\{1,2\}$. The next theorem shows that this definition completely characterises the subspaces of trajectories that may be associated with the implicit form (6) under static local-state feedback control, given controlled-invariant valued boundary conditions.

Theorem 3.3 A subspace $\mathcal{W}$ is controlled-invariant of feedback type for $\Sigma$ if, and only if, there exists an $F \in \mathbb{R}^{m \times n}$ such that (6), with $u_{i, j}=F x_{i, j}$, admits a $\mathcal{W}$-valued trajectory for arbitrary $\mathcal{W}$-valued boundary condition.

Proof: Necessity is established first. Since $A_{0} \mathcal{W} \subseteq E \mathcal{W}+\operatorname{im} B$, two matrices $X_{0}$ and $\Omega$ exist such that $A_{0} W=E W X_{0}+B \Omega$, where $W$ is a basis of $\mathcal{W}$; i.e., im $W=\mathcal{W}$ and $\operatorname{ker} W=\{0\}$. Since $A_{i} \mathcal{W} \subseteq E \mathcal{W}$, for $i \in\{1,2\}$, two matrices $X_{1}$ and $X_{2}$ exist such that $A_{1} W=E W X_{1}$ and $A_{2} W=E W X_{2}$. Since $W$ is of full column-rank, the linear equation $\Omega=-F W$ can be solved in $F$. With its solution we get $\left(A_{0}+B F\right) W=E W X_{0}$. Taking this $F$, the closed-loop system (16) is such that, if $x_{i, j}, x_{i+1, j}$ and $x_{i, j+1}$ are in $\mathcal{W}$, then we can always find $\xi_{i, j}, \xi_{i+1, j}$ and $\xi_{i, j+1}$ for which $x_{i, j}=W \xi_{i, j}, x_{i+1, j}=W \xi_{i+1, j}, x_{i, j+1}=W \xi_{i, j+1}$. Therefore,

$$
\begin{aligned}
A_{0} x_{i, j}+A_{1} x_{i+1, j}+A_{2} x_{i, j+1}+B F x_{i, j} & =\left(A_{0}+B F\right) W \xi_{i, j}+A_{1} W \xi_{i+1, j}+A_{2} W \xi_{i, j+1} \\
& =E W\left(X_{0} \xi_{i, j}+X_{1} \xi_{i+1, j}+X_{2} \xi_{i, j+1}\right) .
\end{aligned}
$$

Defining $x_{i+1, j+1}=W\left(X_{0} \xi_{i, j}+X_{1} \xi_{i+1, j}+X_{2} \xi_{i, j+1}\right)$, and continuing recursively, yields a $\mathcal{W}$-valued trajectory of (6) under the feedback control law $u_{i, j}=F x_{i, j}$.

We now turn to sufficiency. By virtue of (16), the inclusion $\left[\begin{array}{lll}A_{0}+B F & A_{1} & A_{2}\end{array}\right] \bigoplus_{k=1}^{3} \mathcal{W} \subseteq E \mathcal{W}$ must hold, otherwise it would be possible to find $x_{0,0}, x_{1,0}, x_{0,1} \in \mathcal{W}$ such that a $\mathcal{W}$-valued vector $x_{1,1}$ which satisfies $E x_{1,1}=\left(A_{0}+B F\right) x_{0,0}+A_{1} x_{1,0}+A_{2} x_{0,1}$ does not exist. This inclusion implies $\left(A_{0}+B F\right) \mathcal{W} \subseteq E \mathcal{W}$ and $A_{i} \mathcal{W} \subseteq E \mathcal{W}$ for $i \in\{1,2\}$

Given $\mathcal{W}$, a controlled invariant subspace of feedback type for $\Sigma$, any feedback matrix $F \in \mathbb{R}^{m \times n}$ such that $u_{i, j}=F x_{i, j}$ yields a $\mathcal{W}$-valued trajectory for arbitrary $\mathcal{W}$-valued boundary condition is called friend of $\mathcal{W}$.

Theorem 3.4 The set of friends of the controlled invariant subspace of feedback type $\mathcal{W}$, with basis matrix $W$, coincides with the set of matrices $F$ such that $\Omega=-F W$, where $\Omega$ is a solution of $A_{0} W=E W X_{0}+B \Omega$ for some matrix $X_{0}$.

Proof: Let $F$ be such that $\Omega=-F W$, where $\Omega$ is a solution of $A_{0} W=E W X_{0}+B \Omega$ for a certain $X_{0}$. Therefore, $A_{0} W=E W X_{0}-B F W$. Moreover, $A_{1} W=E W X_{1}$ and $A_{2} W=E W X_{2}$. Therefore, $F$ is a friend of $\mathcal{W}$. Conversely, let $F$ be a friend of $\mathcal{W}$. Then, $\left(A_{0}+B F\right) \mathcal{W} \subseteq E \mathcal{W}$ can 
be written as $\left(A_{0}+B F\right) W=E W \Xi$ for a suitable $\Xi$. Hence, $A_{0} W=E W X_{0}+B \Omega$ holds with $X_{0}=\Xi$ and $\Omega=-F W$.

Two degrees of freedom can be recognised in the choice of a friend $F$. The first follows from the the solution $\left(X_{0}, \Omega\right)$ of $A_{0} W=E W X_{0}+B \Omega$. In fact, the set of solutions $\left(X_{0}, \Omega\right)$ is given by

$$
\left[\begin{array}{c}
X_{0} \\
\Omega
\end{array}\right]=\left[\begin{array}{ll}
E W & B
\end{array}\right]^{\dagger} A_{0} W+\left[\begin{array}{c}
H_{0} \\
H_{1}
\end{array}\right] K_{1}
$$

where $\left[\begin{array}{l}H_{0} \\ H_{1}\end{array}\right]$ is a basis matrix of the subspace $\operatorname{ker}\left[\begin{array}{ll}E W & B\end{array}\right]$, and $K_{1}$ is an arbitrary matrix of suitable size. The second degree of freedom comes from the solution of the linear equation $\Omega=-F W$, which can be written as $F=-\Omega\left(W^{\mathrm{T}} W\right)^{-1} W^{\mathrm{T}}+K_{2} Z$, where $Z^{\mathrm{T}}$ is a basis of ker $W^{\mathrm{T}}$ and $K_{2}$ is another arbitrary matrix of suitable size. Thus, as for the 1-D case, there are two degrees of freedom in the computation of a friend $F$; these are given by $K_{1}$ and $K_{2}$.

\subsection{Output-Nulling Subspaces of Feedback Type}

The notion of controlled invariance of feedback type can be extended to output-nulling subspaces. A subspace $\mathcal{W}$ of $\mathcal{X}$ is output-nulling of feedback type if a control input exists such that (6-7), with $\mathfrak{B}_{0}$ boundary condition, admit a solution where the output is identically equal to zero.

Definition 3.4 A subspace $\mathcal{W}$ of $\mathcal{X}$ is output-nulling of feedback type for $\Sigma$ if

- $\left[\begin{array}{c}A_{0} \\ C\end{array}\right] \mathcal{W} \subseteq(E \mathcal{W} \oplus\{0\})+\operatorname{im}\left[\begin{array}{c}B \\ D\end{array}\right]$;

- $A_{1} \mathcal{W}+A_{2} \mathcal{W} \subseteq E \mathcal{W}$.

In view of this definition, note that for strictly proper systems an output-nulling subspace of feedback type is simply a controlled invariant subspace of feedback type contained in the $\operatorname{ker} C$. The next theorem shows that the definition completely characterises the subspaces of trajectories that may be associated with the implicit form (6) under static local-state feedback control, given output-nulling valued boundary conditions.

Theorem 3.5 A subspace $\mathcal{W}$ is output-nulling of feedback type for $\Sigma$ if, and only if, there exists a static local-state feedback control law $u_{i, j}=F x_{i, j}$ such that (6-7) admits a $\mathcal{W}$-valued solution for which the output is identically zero for arbitrary $\mathcal{W}$-valued boundary condition.

The proof can be carried out following the same argument as the proof of Theorem 3.3. Observe in particular that the inclusion $\left[\begin{array}{c}A_{0} \\ C\end{array}\right] \mathcal{W} \subseteq(E \mathcal{W} \oplus\{0\})+\operatorname{im}\left[\begin{array}{l}B \\ D\end{array}\right]$ is equivalent to the existence of two matrices $X_{0}$ and $\Omega$ such that

$$
\left[\begin{array}{c}
A_{0} \\
C
\end{array}\right] W=\left[\begin{array}{c}
E W \\
O
\end{array}\right] X_{0}+\left[\begin{array}{c}
B \\
D
\end{array}\right] \Omega
$$


where $W$ is a basis matrix of $\mathcal{W}$, while as aforementioned in the proof of Theorem 3.3, the inclusion $A_{1} \mathcal{W}+A_{2} \mathcal{W} \subseteq E \mathcal{W}$ is equivalent to the existence of matrices $X_{1}, X_{2}$ such that $A_{i} W=E W X_{i}$ $(i \in\{1,2\})$. Let $F$ be such that $\Omega=-F W$, so that

$$
\left[\begin{array}{c}
A_{0}+B F \\
C+D F
\end{array}\right] W=\left[\begin{array}{c}
E W \\
O
\end{array}\right] X_{0}
$$

It also turns out that an equivalent condition to the two given in Definition 3.4 is the existence of a feedback matrix $F$ such that

$$
\left[\begin{array}{ccc}
A_{0}+B F & A_{1} & A_{2} \\
C+D F & O & O
\end{array}\right] \bigoplus_{k=1}^{3} \mathcal{W} \subseteq E \mathcal{W} \oplus\{0\} .
$$

The set of output-nulling subspaces of feedback type, denoted by $\mathcal{W}(\Sigma)$, is closed under addition. Hence, the maximum output-nulling subspace $\mathcal{W}^{\star}$ of feedback type can still be defined as the sum of all the elements of $\mathcal{W}(\Sigma)$. An algorithm for the computation of $\mathcal{W}^{\star}$, that can be derived by adapting the one for $\mathcal{V}^{\star}$, is given as follows.

Lemma 3.2 $\mathcal{W}^{\star}$ is the last term of the monotonically non-increasing sequence of subspaces $\left\{\mathcal{W}_{i}\right\}_{i}$ given by

$$
\left\{\begin{array}{l}
\mathcal{W}_{0}=\mathcal{X} \\
\mathcal{W}_{i}=\left[\begin{array}{c}
A_{0} \\
C
\end{array}\right]^{-1}\left(\left(E \mathcal{W}_{i-1} \oplus\{0\}\right)+\mathrm{im}\left[\begin{array}{l}
B \\
D
\end{array}\right]\right) \cap\left[\begin{array}{l}
A_{1} \\
A_{2}
\end{array}\right]^{-1}\left(E \mathcal{W}_{i-1} \oplus E \mathcal{W}_{i-1}\right),
\end{array}\right.
$$

with $i \in\{1,2, \ldots, k\}$, where the integer $k \leq n-1$ is determined by the condition $\mathcal{W}_{k+1}=\mathcal{W}_{k}$, i.e., $\mathcal{W}^{\star}=\mathcal{W}_{k}$.

The proof follows from that of Lemma 3.1 with the obvious modifications.

Example 3.1 We show that an output-nulling subspace is not necessarily of feedback type. Consider a system $\Sigma$ described by the matrices

$$
\begin{aligned}
& E=\left[\begin{array}{lll}
0 & 0 & 0 \\
0 & 0 & 0
\end{array}\right], A_{0}=\left[\begin{array}{ccc}
3 & 0 & -2 \\
2 & 0 & 0
\end{array}\right], A_{1}=\left[\begin{array}{ccc}
0 & 0 & -2 \\
-3 & 0 & 0
\end{array}\right], A_{2}=\left[\begin{array}{ccc}
0 & 0 & -4 \\
-3 & 0 & 0
\end{array}\right], B=\left[\begin{array}{cc}
0 & 1 \\
-1 & -4
\end{array}\right], \\
& C=\left[\begin{array}{lll}
-4 & 0 & 0
\end{array}\right], D=0 .
\end{aligned}
$$

It is easy to verify that the sequence of subspaces $\left\{\mathcal{V}_{i}\right\}_{i}$ in Lemma 3.1 converges in a single step to the subspace $\mathcal{V}^{\star}=\left[\begin{array}{ll}0 & 0 \\ 1 & 0 \\ 0 & 1\end{array}\right]$, whereas the sequence $\left\{\mathcal{W}_{i}\right\}_{i}$ in Lemma 3.2 converges in one step to the subspace $\mathcal{W}^{\star}=\left[\begin{array}{l}0 \\ 1 \\ 0\end{array}\right]$. 


\subsection{Disturbance Decoupling Problem (DDP)}

The notion of output-nulling subspace of feedback type is useful in the solution of the DDP problem. Consider the model

$$
\begin{aligned}
E x_{i+1, j+1} & =A_{0} x_{i, j}+A_{1} x_{i+1, j}+A_{2} x_{i, j+1}+B u_{i, j}+H w_{i, j}, \\
y_{i, j} & =C x_{i, j}+D u_{i, j}+G w_{i, j}
\end{aligned}
$$

where $w_{i, j}$ is a disturbance to be rejected using a control $u_{i, j}=F x_{i, j}$. That is the aim is to find $F \in \mathbb{R}^{m \times n}$ such that for zero-valued boundary conditions the closed-loop system

$$
\begin{aligned}
E x_{i+1, j+1} & =\left(A_{0}+B F\right) x_{i, j}+A_{1} x_{i+1, j}+A_{2} x_{i, j+1}+H w_{i, j}, \\
y_{i, j} & =(C+D F) x_{i, j}+G w_{i, j},
\end{aligned}
$$

admits a trajectory with an output that is not affected by the disturbance $w$; i.e., such that the corresponding $y_{i, j}$ is zero for all $(i, j) \in \mathfrak{B}$. The notion of controlled invariance proposed in [6] led to a sufficient condition for the solution of the DDP. In the next theorem, we show that the notion of output-nulling subspace of feedback type developed above, leads to a necessary and sufficient condition for the solvability of this problem. In other words, the DDP problem is solved here without conservatism for the first time within the context of implicit FM models for 2-D systems.

Theorem 3.6 The DDP admits solutions if, and only if,

$$
\operatorname{im}\left[\begin{array}{l}
H \\
G
\end{array}\right] \subseteq E \mathcal{W}^{\star} \oplus\{0\},
$$

Equivalently, $\operatorname{im} H \subseteq E \mathcal{W}^{\star}$ and $G=0$.

Proof: (If). By taking $F$ to be a friend of $\mathcal{W}^{\star}$ and $G=0$, for each $\mathcal{W}^{\star}$-valued boundary condition, a solution of $(20)$ lies on $\mathcal{W}^{\star}$ for all $(i, j) \in \mathfrak{B}$, and therefore the system is disturbance decoupled.

(Only if). Suppose the closed-loop system is disturbance decoupled, i.e., $F$ exists such that $y_{i, j}=0$ for any disturbance $w_{i, j}$. When $w_{i, j}$ is zero, the closed-loop system is still disturbance decoupled, i.e., $x_{i, j}$ lies for all $(i, j)$ on the largest subspace $\mathcal{T}$ satisfying

$$
\left[\begin{array}{ccc}
A_{0}+B F & A_{1} & A_{2} \\
C+D F & O & O
\end{array}\right] \bigoplus_{k=1}^{3} \mathcal{T} \subseteq E \mathcal{T} .
$$

By definition, this subspace is indeed $\mathcal{W}^{\star}$. Moreover, since the system must be decoupled for each value of $w_{i, j}, H$ must satisfy im $H \subseteq E \mathcal{T}=E \mathcal{W}^{\star}$.

Remark 3.3 The solution of the disturbance decoupling problem can easily be extended to the case where the disturbance to reject is measurable, so that the control can be expressed as $u_{i, j}=$ 
$F x_{i, j}+S w_{i, j}$. The problem is to find matrices $F$ and $S$ such that the output of the closed-loop system

$$
\begin{aligned}
E x_{i+1, j+1} & =\left(A_{0}+B F\right) x_{i, j}+A_{1} x_{i+1, j}+A_{2} x_{i, j+1}+B S w_{i, j}+H w_{i, j} \\
y_{i, j} & =(C+D F) x_{i, j}+D S w_{i, j}+G w_{i, j}
\end{aligned}
$$

is zero for zero boundary conditions and for any disturbance $w_{i, j}$. Using the same arguments of Theorem 3.6, we can show that the problem is solvable if and only if

$$
\operatorname{im}\left[\begin{array}{l}
H \\
G
\end{array}\right] \subseteq\left(E \mathcal{W}^{\star} \oplus\{0\}\right)+\operatorname{im}\left[\begin{array}{l}
B \\
D
\end{array}\right]
$$

In fact, if this condition holds, $\left[\begin{array}{l}H \\ G\end{array}\right]$ can be decomposed as

$$
\left[\begin{array}{l}
H \\
G
\end{array}\right]=\left[\begin{array}{l}
H_{1} \\
G_{1}
\end{array}\right] \Phi+\left[\begin{array}{l}
H_{2} \\
G_{2}
\end{array}\right] \Psi
$$

where

$$
\operatorname{im}\left[\begin{array}{l}
H_{1} \\
G_{1}
\end{array}\right] \subseteq E \mathcal{W}^{\star} \oplus\{0\} \quad \text { and } \quad \operatorname{im}\left[\begin{array}{c}
H_{2} \\
G_{2}
\end{array}\right] \subseteq \operatorname{im}\left[\begin{array}{c}
B \\
D
\end{array}\right]
$$

The closed-loop system becomes

$$
\begin{aligned}
E x_{i+1, j+1} & =\left(A_{0}+B F\right) x_{i, j}+A_{1} x_{i+1, j}+A_{2} x_{i, j+1}+\left(B S+H_{2}\right) w_{i, j}+H_{1} w_{i, j}, \\
y_{i, j} & =(C+D F) x_{i, j}+\left(D S+G_{2}\right) w_{i, j},
\end{aligned}
$$

By choosing $S$ to satisfy $\left[\begin{array}{l}B \\ D\end{array}\right] S+\left[\begin{array}{l}H_{2} \\ G_{2}\end{array}\right]=0-$ which is possible since im $\left[\begin{array}{l}H_{2} \\ G_{2}\end{array}\right] \subseteq \operatorname{im}\left[\begin{array}{l}B \\ D\end{array}\right]-$ and $F$ to be a friend of $\mathcal{W}^{\star}$, given a $\mathcal{W}^{\star}$-valued boundary condition, the local state remains on $\mathcal{W}^{\star}$, and $y_{i, j}$ is zero.

\section{Duality}

In this section we present the dualisation of the concepts introduced in the previous sections. First, the dual concept of controlled invariance is called conditioned invariance. While controlled invariant subspaces reside in the latent variable space $\mathcal{X}$, their duals lie in the outer space $\underline{\mathcal{X}}$.

Definition 4.1 A subspace $\underline{\mathcal{S}}$ of the outer space $\underline{\mathcal{X}}$ is conditioned invariant for $\Sigma$ if

$$
A_{i}\left(E^{-1} \underline{\mathcal{S}} \cap \operatorname{ker} C\right) \subseteq \underline{\mathcal{S}}, \quad i \in\{0,1,2\}
$$

The duality between 2-D controlled and conditioned invariance can be stated in precise terms as follows. Let $\Sigma^{\mathrm{T}}$ identify the dual system of $(6-7)$, i.e., $\Sigma^{\mathrm{T}} \stackrel{\text { def }}{=}\left(E^{\mathrm{T}} ; A_{0}^{\mathrm{T}}, A_{1}^{\mathrm{T}}, A_{2}^{\mathrm{T}} ; C^{\mathrm{T}} ; B^{\mathrm{T}} ; D^{\mathrm{T}}\right)$.

Lemma 4.1 The orthogonal complement of a controlled invariant for $\Sigma$ is conditioned invariant for $\Sigma^{T}$, and vice-versa. 
Proof: Let $H \in \mathbb{R}^{n_{2} \times n_{1}}$ identify a linear map between $\mathbb{R}^{n_{1}}$ and $\mathbb{R}^{n_{2}}$. Let $\mathcal{A}$ and $\mathcal{B}$ be subspaces of $\mathbb{R}^{n_{1}}$, and let $\mathcal{C}$ be a subspace of $\mathbb{R}^{n_{2}}$. Using the well-known identities $[2$, p. 326]

$$
\begin{aligned}
(\mathcal{A}+\mathcal{B})^{\perp} & =\mathcal{A}^{\perp} \cap \mathcal{B}^{\perp} \\
(\mathcal{A} \cap \mathcal{B})^{\perp} & =\mathcal{A}^{\perp}+\mathcal{B}^{\perp} \\
H(\mathcal{A}+\mathcal{B}) & =H \mathcal{A}+H \mathcal{B} \\
\left(H^{-1} \mathcal{C}\right)^{\perp} & =H^{\mathrm{T}} \mathcal{C}^{\perp} \\
H \mathcal{A} \subseteq \mathcal{C} & \Leftrightarrow H^{\mathrm{T}} \mathcal{C}^{\perp} \subseteq \mathcal{A}^{\perp}
\end{aligned}
$$

it is easily seen that by computing the orthogonal complement of

$$
\sum_{j=0}^{2} A_{j} \mathcal{V} \subseteq E \mathcal{V}+\operatorname{im} B
$$

we obtain

$$
\bigcap_{j=0}^{2} A_{j}^{\mathrm{T}}\left(E^{-\mathrm{T}} \mathcal{V}^{\perp} \cap \operatorname{ker} B^{\mathrm{T}}\right) \subseteq \mathcal{V}^{\perp}
$$

which says that $\mathcal{V}^{\perp}$ is conditioned invariant for $\Sigma^{\mathrm{T}}$.

The duals of 2-D output-nulling subspaces are the 2-D input-containing subspaces. When $D=0$, an input-containing subspace $\underline{\mathcal{S}}$ is a conditioned invariant subspace of $\Sigma$ that contains $\operatorname{im} B$. By dualising the definition of output-nulling subspace we see that an input-containing subspace $\underline{\mathcal{S}}$ is a subspace that satisfies the subspace inclusion

$$
\left[\begin{array}{ll}
A_{0} & B \\
A_{1} & O \\
A_{2} & O
\end{array}\right]\left(\left(E^{-1} \underline{\mathcal{S}} \oplus \mathcal{U}\right) \cap \operatorname{ker}\left[\begin{array}{ll}
C & D
\end{array}\right]\right) \subseteq \bigoplus_{k=0}^{3} \underline{\mathcal{S}} .
$$

The set of input-containing subspaces of $\Sigma$ is denoted by $\mathcal{S}(\Sigma)$. As for the set of 2-D conditioned invariant subspaces, this set is closed under subspace intersection but not under subspace addition. Therefore, $(\mathcal{S}(\Sigma), \cap ; \subseteq)$ is a (non-distributive and modular) lower semi-lattice with respect to the binary operation $\cap$ and with respect to the partial ordering $\subseteq$. Thus, it admits a minimum given by $\mathcal{S}^{\star}=\min \mathcal{S}(\Sigma)=\bigcap_{\mathcal{S} \in \mathcal{S}(\Sigma)} \mathcal{S}$. By dualising the algorithm for $\mathcal{V}^{\star}$, we have the following.

Lemma $4.2 \mathcal{S}^{\star}$ is the last term of the monotonically non-decreasing sequence of subspaces $\left\{\underline{\mathcal{S}}_{i}\right\}_{i}$ given by

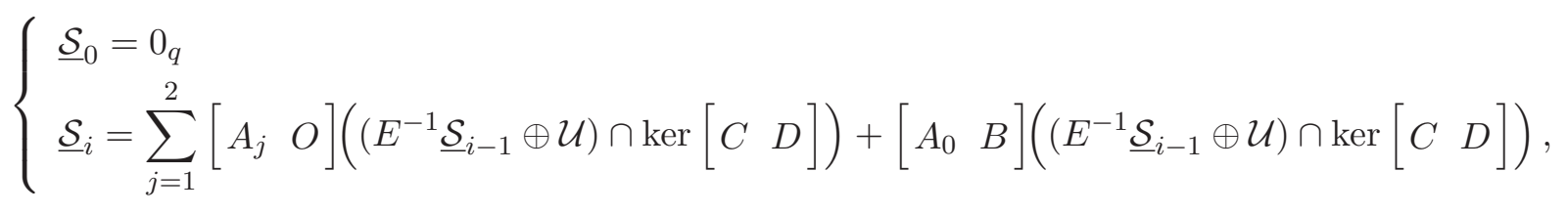

where $i \in\{1,2, \ldots, k\}$, and where the integer $k \leq n-1$ is determined by the condition $\underline{\mathcal{S}}_{k+1}=\underline{\mathcal{S}}_{k}$, i.e., $\underline{\mathcal{S}}_{k}=\underline{\mathcal{S}}^{\star}$. 
Similarly to $[14,13]$, we can also define the inner 2-D conditioned invariant subspaces from the sequence $\left\{\mathcal{S}_{i}\right\}_{i}$ given by

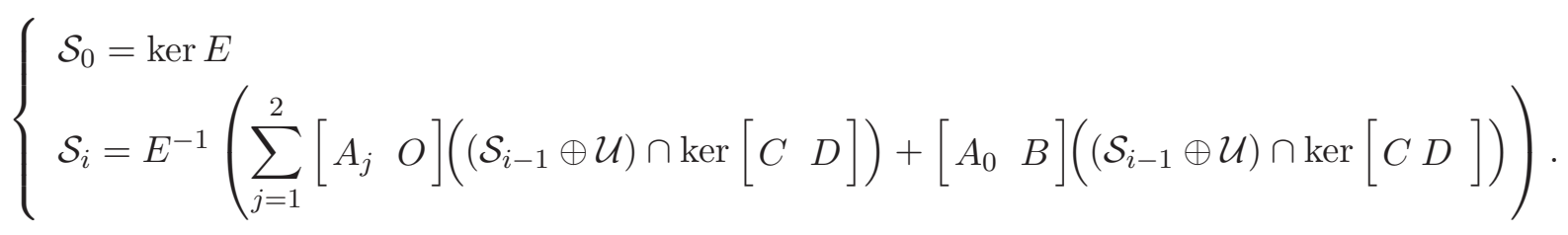

This definition guarantees that $\mathcal{S}_{i}=E^{-1} \underline{\mathcal{S}}_{i}$ for all $i \in \mathbb{N}$. The duals of inner 2-D conditioned invariant subspaces will be called outer 2-D controlled invariant subspaces. These are defined via the recursion $\left\{\underline{\mathcal{V}}_{i}\right\}_{i}$ given by

$$
\left\{\begin{array}{l}
\underline{\mathcal{V}}_{0}=\operatorname{im} E \\
\underline{\mathcal{V}}_{i}=E\left(\bigcap_{j=1}^{2}\left[\begin{array}{c}
A_{j} \\
O
\end{array}\right]^{-1}\left(\left(\underline{\mathcal{V}}_{i-1} \oplus\{0\}\right)+\operatorname{im}\left[\begin{array}{c}
B \\
D
\end{array}\right]\right) \cap\left[\begin{array}{c}
A_{0} \\
C
\end{array}\right]^{-1}\left(\left(\underline{\mathcal{V}}_{i-1} \oplus\{0\}\right)+\operatorname{im}\left[\begin{array}{c}
B \\
D
\end{array}\right]\right)\right) .
\end{array}\right.
$$

There holds $\underline{\mathcal{V}}_{i}=E \mathcal{V}_{i}$ for all $i \in \mathbb{N}$. The relationship between inner and outer subspaces is illustrated in Figure 2.

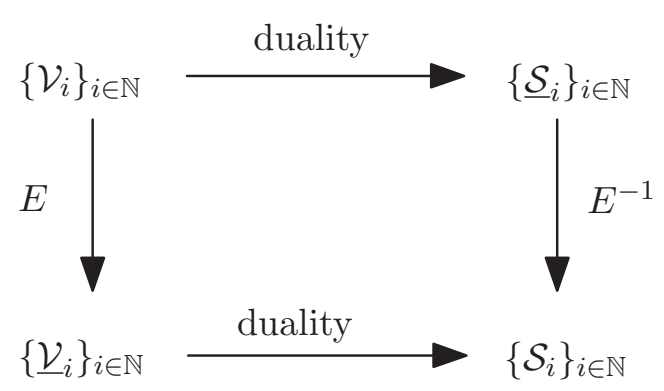

Figure 2: Complete duality between controlled and conditioned invariance.

We now consider the dual of controlled-invariance of feedback type, which is called conditioned invariance of output-injection type.

Definition 4.2 Subspace $\underline{\mathcal{Z}}$ is conditioned-invariant of output-injection type for $\Sigma$ if

- $A_{0}\left(E^{-1} \underline{\mathcal{Z}} \cap \operatorname{ker} C\right) \subseteq \underline{\mathcal{Z}}$;

- $A_{i} E^{-1} \underline{\mathcal{Z}} \subseteq \underline{\mathcal{Z}}$ for $i \in\{1,2\}$.

We show that for conditioned invariant subspaces of output-injection type the existence of a matrix $G$ is guaranteed such that

$$
\left(A_{0}+G C\right) E^{-1} \underline{\mathcal{Z}} \subseteq \underline{\mathcal{Z}}, \quad A_{1} E^{-1} \underline{\mathcal{Z}} \subseteq \underline{\mathcal{Z}}, \quad A_{2} E^{-1} \underline{\mathcal{Z}} \subseteq \underline{\mathcal{Z}} .
$$

Consider $A_{0}\left(E^{-1} \underline{\mathcal{Z}} \cap \operatorname{ker} C\right) \subseteq \underline{\mathcal{Z}}$. This inclusion says that

$$
E^{-1} \operatorname{ker} Q \cap \operatorname{ker} C \subseteq \operatorname{ker}\left(Q A_{0}\right),
$$


where $Q$ is a full row-rank matrix such that $\operatorname{ker} Q=\underline{\mathcal{Z}}$. Hence,

$$
\operatorname{ker}\left[\begin{array}{c}
Q E \\
C
\end{array}\right] \subseteq \operatorname{ker}\left(Q A_{0}\right) \text {. }
$$

As such, two matrices $\Gamma_{0}$ and $\Lambda$ exist such that

$$
Q A_{0}=\left[\begin{array}{ll}
\Gamma_{0} & \Lambda
\end{array}\right]\left[\begin{array}{c}
Q E \\
C
\end{array}\right]
$$

i.e., $Q A_{0}=\Gamma_{0} Q E+\Lambda C$. The output-injection matrix $G$ can be constructed as follows. First, all matrices $\Gamma$ and $\Lambda$ such that (26) hold are given by

$$
\left[\begin{array}{cc}
\Gamma_{0} & \Lambda
\end{array}\right]=Q A_{0}\left[\begin{array}{c}
Q E \\
C
\end{array}\right]^{\dagger}+K_{1} H
$$

where $H$ has linearly independent rows and $\operatorname{ker} H=\operatorname{im}\left[\begin{array}{c}Q E \\ C\end{array}\right]$, while $K_{1}$ is an arbitrary matrix of suitable size. Matrix $K_{1}$ represents a first degree of freedom in the construction of the outputinjection matrix. Then, the set of all output-injection matrices is given by the solution of $\Lambda=-Q G$, which are

$$
G=-Q^{\mathrm{T}}\left(Q Q^{\mathrm{T}}\right)^{-1} \Lambda+\Omega K_{2},
$$

where $\Omega$ is a basis of $\operatorname{ker} Q$ and $K_{2}$ is an arbitrary matrix of suitable size. Hence, $K_{2}$ represents a second degree of freedom in the construction of the output-injection matrix associated with $\underline{\mathcal{Z}}$. Moreover, since $A_{1} E^{-1} \underline{\mathcal{Z}} \subseteq \underline{\mathcal{Z}}$ and $A_{2} E^{-1} \underline{\mathcal{Z}} \subseteq \underline{\mathcal{Z}}$, two matrices $\Gamma_{1}$ and $\Gamma_{2}$ exist such that

$$
Q A_{i}=\Gamma_{i} Q E
$$

The dual of an output-nulling subspace of feedback type is an input-containing subspace of outputinjection type.

Definition 4.3 Subspace $\underline{\mathcal{Z}}$ is input containing of output-injection type for $\Sigma$ if

- $\left[\begin{array}{ll}A_{0} & B\end{array}\right]\left(\left(E^{-1} \underline{\mathcal{Z}} \oplus \mathcal{U}\right) \cap \operatorname{ker}\left[\begin{array}{ll}C & D\end{array}\right]\right) \subseteq \underline{\mathcal{Z}}$

- $A_{i} E^{-1} \underline{\mathcal{Z}} \subseteq \underline{\mathcal{Z}}$ for $i \in\{1,2\}$.

Dualising the algorithm for the computation of the largest output-nulling subspace of feedback type $\mathcal{W}^{\star}$, we obtain an algorithm for $\underline{\mathcal{Z}}^{\star}$.

Lemma $4.3 \underline{\mathcal{Z}}^{\star}$ is the last term of the monotonically non-increasing sequence of subspaces $\left\{\mathcal{Z}_{i}\right\}_{i}$ given by

$$
\left\{\begin{array}{l}
\underline{\mathcal{Z}}_{0}=0 \\
\underline{\mathcal{Z}}_{i}=\left[\begin{array}{ll}
A_{0} & B
\end{array}\right]\left(\left(E^{-1} \underline{\mathcal{Z}}_{i-1} \oplus \mathcal{U}\right) \cap \operatorname{ker}\left[\begin{array}{ll}
C & D
\end{array}\right]\right)+\left[\begin{array}{ll}
A_{1} & A_{2}
\end{array}\right]\left(E^{-1} \underline{\mathcal{Z}}_{i-1} \oplus E^{-1} \underline{\mathcal{Z}}_{i-1}\right),
\end{array}\right.
$$

with $i \in\{1,2, \ldots, k\}$, where the integer $k \leq n-1$ is determined by the condition $\underline{\mathcal{Z}}_{k+1}=\underline{\mathcal{Z}}_{k}$, i.e., $\underline{\mathcal{Z}}^{\star}=\underline{\mathcal{Z}}_{k}$. 
Consider $\left[\begin{array}{ll}A_{0} & B\end{array}\right]\left(\left(E^{-1} \underline{\mathcal{Z}} \oplus \mathcal{U}\right) \cap \operatorname{ker}\left[\begin{array}{ll}C & D\end{array}\right]\right) \subseteq \underline{\mathcal{Z}}$. This inclusion says that

$$
\left(E^{-1} \operatorname{ker} Q \oplus \mathcal{U}\right) \cap \operatorname{ker}\left[\begin{array}{ll}
C & D
\end{array}\right] \subseteq \operatorname{ker}\left(Q\left[\begin{array}{ll}
A_{0} & B
\end{array}\right]\right),
$$

where $Q$ is a full row-rank matrix such that $\operatorname{ker} Q=\underline{\mathcal{Z}}$. Hence,

$$
\operatorname{ker}\left[\begin{array}{cc}
Q E & O \\
C & D
\end{array}\right] \subseteq \operatorname{ker}\left(Q\left[\begin{array}{ll}
A_{0} & B
\end{array}\right]\right) \text {. }
$$

As such, two matrices $\Gamma_{0}$ and $\Lambda$ exist such that

$$
Q\left[\begin{array}{ll}
A_{0} & B
\end{array}\right]=\left[\begin{array}{ll}
\Gamma_{0} & \Lambda
\end{array}\right]\left[\begin{array}{cc}
Q E & O \\
C & D
\end{array}\right]
$$

The relationship between inner and outer subspaces is illustrated in Figure 3.

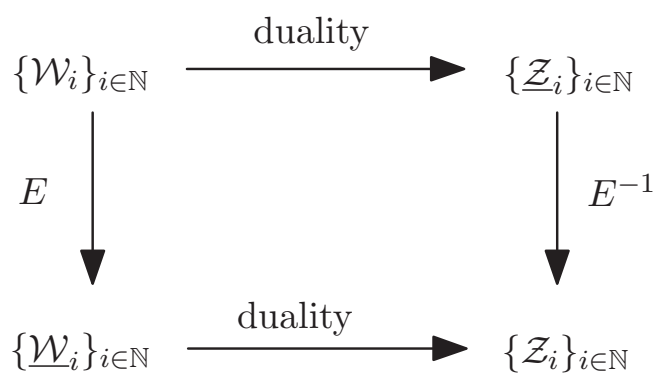

Figure 3: Complete duality between output-nulling and input-containing subspaces.

Now we relate the concept of conditioned invariance of output-injection type with the existence of certain local state reconstructors that maintain information on the local state of $\Sigma$ modulo a certain subspace, which is an inner conditioned invariant subspace. More precisely, given the subspace $\underline{\mathcal{Z}}$ and a full row-rank matrix $Q$ such that $\underline{\mathcal{Z}}=\operatorname{ker} Q$, we define (with a slight abuse of nomenclature) a $\underline{\mathcal{Z}}$-quotient observer, as it is done in [18], for (6-7) as a system ruled by

$$
\omega_{i+1, j+1}=K_{0} \omega_{i, j}+K_{1} \omega_{i+1, j}+K_{2} \omega_{i, j+1}+L y_{i, j}
$$

such that if $\omega_{i, j}=Q E x_{i, j}$ for all $(i, j) \in \mathfrak{B}_{0}$, then $\omega_{i, j}=Q E x_{i, j}$ for all $(i, j) \in \mathfrak{B}$. In other words, a $\underline{\mathcal{Z}}$-quotient observer maintains the knowledge of the components of the local state modulo $\operatorname{ker}(Q E)$. Since $\operatorname{ker}(Q E)=E^{-1} \operatorname{ker} Q=E^{-1} \underline{\mathcal{Z}}$, a $\underline{\mathcal{Z}}$-quotient observer maintains the knowledge of the components of the local state modulo the inner conditioned invariant $E^{-1} \underline{\mathcal{Z}}$. In other words, if $\omega_{i, j}=x_{i, j} /\left(E^{-1} \underline{\mathcal{Z}}\right)$ on the boundary, then $\omega_{i, j}=x_{i, j} /\left(E^{-1} \underline{\mathcal{Z}}\right)$ everywhere. Thus, if the initial conditions of the system and of the quotient observer are equal modulo $\left(E^{-1} \underline{\mathcal{Z}}\right)$, the state of the quotient observer is always equal to the local state of the system modulo $\left(E^{-1} \underline{\mathcal{Z}}\right)$. The 2-D system (28) is here referred to as a $\underline{\mathcal{Z}}$-quotient observer even if as a matter of fact it only maintains information on the local state of $\Sigma$, but it does not reconstruct such information in the case of mismatched boundary conditions of $\Sigma$ and (28). 
The following theorem provides a characterisation of conditioned invariance of output-injection type.

Theorem 4.1 A subspace $\underline{\mathcal{Z}}$ is an input-containing subspace of output-injection type for $\Sigma$ if and only if there exists a $\underline{\mathcal{Z}}$-quotient observer for $\Sigma$.

Proof: (Only if). Since $\underline{\mathcal{Z}}$ is an input-containing subspace of output-injection type for $\Sigma$, we can write $\underline{\mathcal{Z}}=\operatorname{ker} Q$, where $Q$ satisfies the linear equations

$$
\begin{aligned}
& Q A_{0}=\Gamma_{0} Q E+\Lambda C, \quad Q A_{1}=\Gamma_{1} Q E, \\
& Q A_{2}=\Gamma_{2} Q E, \quad Q B=\Lambda D .
\end{aligned}
$$

Consider (28) with $K_{i}=\Gamma_{i}$ for $i \in\{0,1,2\}$, and $L=\Lambda$. Define the error as $e_{i, j} \stackrel{\text { def }}{=} Q E x_{i, j}-\omega_{i, j}$. Since it is assumed that $\omega_{i, j}=Q E x_{i, j}$ over the boundary $\mathfrak{B}_{0}, e_{i, j}=0$ for all $(i, j) \in \mathfrak{B}_{0}$. Thus,

$$
\begin{aligned}
e_{i+1, j+1}= & Q E x_{i+1, j+1}-\omega_{i+1, j+1} \\
= & Q A_{0} x_{i, j}+Q A_{1} x_{i+1, j}+Q A_{2} x_{i, j+1}+Q B u_{i, j} \\
& -\Gamma_{0} \omega_{i, j}-\Gamma_{1} \omega_{i+1, j}-\Gamma_{2} \omega_{i, j+1}-\Lambda C x_{i, j}-\Lambda D u_{i, j} \\
= & \Gamma_{0} e_{i, j}+\Gamma_{1} e_{i+1, j}+\Gamma_{2} e_{i, j+1} .
\end{aligned}
$$

Since these dynamics are autonomous, the error is zero everywhere if it is zero over $\mathfrak{B}_{0}$.

(If). Suppose a $\underline{\mathcal{Z}}$-quotient observer for $\Sigma$ exists. Therefore, given $\omega_{i, j}=Q E x_{i, j}$ over the boundary $\mathfrak{B}_{0}$, we have $\omega_{i, j}=Q E x_{i, j}$ over $\mathfrak{B}$. Let the boundary condition of (6) be such that for a certain $u_{0,0} \in \mathcal{U}$ we have $\left[\begin{array}{l}x_{0,0} \\ u_{0,0}\end{array}\right] \in \operatorname{ker}\left[\begin{array}{cc}Q E & O \\ C & D\end{array}\right]$ and $x_{1,0}, x_{0,1} \in \operatorname{ker}(Q E)$. The boundary condition of the $\underline{\mathcal{Z}}$-quotient observer is such that $\omega_{0,0}=\omega_{1,0}=\omega_{0,1}=0$. This is compatible with the fact that $\omega_{i, j}=Q E x_{i, j}$ for $(i, j) \in\{(0,0),(1,0),(0,1)\}$, since for such pairs of indexes we have $x_{i, j} \in \underline{\mathcal{Z}}$, and hence $Q E x_{i, j}=0$. Therefore, from (28) it is found that

$$
\omega_{1,1}=K_{0} \omega_{0,0}+K_{1} \omega_{1,0}+K_{2} \omega_{0,1}+L C x_{0,0}+L D u_{0,0}
$$

which is zero since $C x_{0,0}+D u_{0,0}=0$. On the other hand, $E x_{1,1}=A_{0} x_{0,0}+A_{1} x_{1,0}+A_{2} x_{0,1}+B u_{0,0}$ leads to

$$
Q E x_{1,1}=Q\left[\begin{array}{ll}
A_{0} & B
\end{array}\right]\left[\begin{array}{l}
x_{0,0} \\
u_{0,0}
\end{array}\right]+Q A_{1} x_{1,0}+Q A_{2} x_{0,1}=\omega_{1,1},
$$

which is zero as shown above. For the arbitrariness of $x_{0,0}, x_{1,0}, x_{0,1}$ and $u_{0,0}$ we get $Q\left[\begin{array}{ll}A_{0} & B\end{array}\right] \operatorname{ker}\left[\begin{array}{ccc}Q E & O \\ C & D\end{array}\right]=$ $\{0\}$ and $Q A_{i} \operatorname{ker}(Q E)=\{0\}$ for $i \in\{1,2\}$. Hence, $\underline{\mathcal{Z}}$ is an input-containing subspace of outputinjection type.

\section{Conclusions}

Structural invariants of an implicit 2-D model are identified and studied in this paper, within the context of quater-plane causal solutions for compatible boundary conditions on the local state. Di- 
rections for future work include extensions of the main ideas to the study of non-causal solutions for plurilateral boundary conditions.

\section{References}

[1] G. Basile and G. Marro, Controlled and conditioned invariant subspaces in linear system theory, Journal of Optimization Theory and Applications, 3(5):306-315, May 1969.

[2] G. Basile and G. Marro, Controlled and Conditioned Invariants in Linear System Theory. Prentice Hall, New Jersey, 1992.

[3] P. Bernhard, On singular implicit linear dynamical systems, SIAM Journal of Control and Optimization, 20(5):612-633, 1982.

[4] G. Conte and A. Perdon, On the geometry of 2D systems, Proceedings of the IEEE International Symposium on Circuit and Systems, Helsinki, 1988.

[5] G. Conte and A. Perdon, A geometric approach to the theory of 2-D systems, IEEE Transactions on Automatic Control, AC-33:946-950, 1988.

[6] G. Conte, A. Perdon, and T. Kaczorek, Geometric methods in the theory of singular 2D linear systems, Kybernetika, vol. 27:263-270, 1991.

[7] T. Kaczorek, $(A, B)$-invariant subspaces and $\mathcal{V}$-invariant subspaces for Fornasini-Marchesini's model, Bulletin of the Polish Academy of Sciences, Technical Sciences, 35, 1988.

[8] T. Kaczorek. Singular general model of 2D systems and its solutions. IEEE Transactions on Automatic Control, AC-33:1060-1061, 1988.

[9] E. Fornasini and G. Marchesini, State-space realization theory of two-dimensional filters, IEEE Transactions on Automatic Control, AC-21, no. 4, pp. 484-492, August 1976.

[10] E. Fornasini and G. Marchesini, Doubly-Indexed Dynamical Systems: State-Space Models and Structural Properties, Mathematical Systems Theory, vol. 12, pp. 59-72, 1978.

[11] M.L.J. Hautus, Controlled invariance in sytems over rings, Springer Lecture Notes in Control and Info. Sci. 39, 1982.

[12] A. Karamanciog̃lu and F.L. Lewis, Geometric theory for the singular Roesser model, IEEE Transactions on Automatic Control, AC-37(6):801-806, June 1992.

[13] F.L. Lewis, A tutorial on the geometric analysis of linear time-invariant implicit systems, Automatica, vol. 28, pp. 119-137, 1992. 
[14] M. Malabre, Generalized linear systems: geometric and structural approaches, Linear Algebra and Its Applications, vol. 122-124, pp. 591-621, 1989.

[15] A. Morse, Structural invariants of linear multivariable systems," SIAM Journal of Control, 11(3), pp. 446-465, 1973.

[16] L. Ntogramatzidis, Self-bounded output-nulling subspaces for non strictly proper systems and their application to the disturbance decoupling problem, IEEE Transactions on Automatic Control, vol. 53, no. 1, pp. 423-428, 2008.

[17] L. Ntogramatzidis, Structural invariants of two-dimensional systems, SIAM Journal on Control and Optimization, 50(1): 334-356, 2012.

[18] L. Ntogramatzidis, and M. Cantoni, Detectability subspaces and observer synthesis for twodimensional systems, Multidimensional Systems and Signal Processing, 23:79-96, 2012.

[19] L. Ntogramatzidis, and M. Cantoni, Structural invariants for implicit two-dimensional systems, In Proceedings of the $7^{\text {th }}$ International Workshop on Multidimensional (nD) Systems (NdS 2011), Poitiers, France, September 5-7, 2011.

[20] L. Ntogramatzidis, M. Cantoni, and R. Yang, A geometric theory for 2-D systems including notions of stabilisability, Multidimensional Systems and Signal Processing, 19: 449-475, 2008.

[21] H.L. Trentelman, A.A. Stoorvogel, and M. Hautus. Control theory for linear systems. Communications and Control Engineering. Springer, 2001.

[22] J. Willems, Almost invariant subspaces: an approach to high gain feedback design - Part II: Almost conditionally invariant subspaces, IEEE Transactions on Automatic Control, AC-26(1), pp. 235-252, 1981.

[23] W.M. Wonham. Linear Multivariable Control: A Geometric Approach. Springer-Verlag, 3 edition, 1985.

[24] R. Yang, L. Ntogramatzidis, and M. Cantoni, On the realisation of 2-D linear systems with recursively computable latent variable models, IEEE Transactions on Circuits and Systems - I, 56(3): 644-652, 2009. 


\section{University Library}

\section{- M M N E R VA A gateway to Melbourne's research publications}

Minerva Access is the Institutional Repository of The University of Melbourne

Author/s:

Ntogramatzidis, L;Cantoni, M

Title:

Geometric techniques for implicit two-dimensional systems

Date:

2013-12-01

Citation:

Ntogramatzidis, L. \& Cantoni, M. (2013). Geometric techniques for implicit two-dimensional systems. MULTIDIMENSIONAL SYSTEMS AND SIGNAL PROCESSING, 24 (4), pp.601-620. https://doi.org/10.1007/s11045-012-0205-4.

Persistent Link:

http://hdl.handle.net/11343/283016 